\title{
Semidefinite bounds for nonbinary codes based on quadruples
}

\author{
Bart Litjens $^{1}$ • Sven Polak ${ }^{1}$ • Alexander Schrijver ${ }^{1}$
}

Received: 30 December 2015 / Revised: 21 April 2016 / Accepted: 25 April 2016 /

Published online: 11 May 2016

(C) The Author(s) 2016. This article is published with open access at Springerlink.com

\begin{abstract}
For nonnegative integers $q, n, d$, let $A_{q}(n, d)$ denote the maximum cardinality of a code of length $n$ over an alphabet $[q]$ with $q$ letters and with minimum distance at least $d$. We consider the following upper bound on $A_{q}(n, d)$. For any $k$, let $\mathcal{C}_{k}$ be the collection of codes of cardinality at most $k$. Then $A_{q}(n, d)$ is at most the maximum value of $\sum_{v \in[q]^{n}} x(\{v\})$, where $x$ is a function $\mathcal{C}_{4} \rightarrow \mathbb{R}_{+}$such that $x(\emptyset)=1$ and $x(C)=0$ if $C$ has minimum distance less than $d$, and such that the $\mathcal{C}_{2} \times \mathcal{C}_{2}$ matrix $\left(x\left(C \cup C^{\prime}\right)\right)_{C, C^{\prime} \in \mathcal{C}_{2}}$ is positive semidefinite. By the symmetry of the problem, we can apply representation theory to reduce the problem to a semidefinite programming problem with order bounded by a polynomial in $n$. It yields the new upper bounds $A_{4}(6,3) \leq 176, A_{4}(7,3) \leq 596, A_{4}(7,4) \leq 155, A_{5}(7,4) \leq 489$, and $A_{5}(7,5) \leq 87$.
\end{abstract}

Keywords Code $\cdot$ Nonbinary code $\cdot$ Upper bounds $\cdot$ Semidefinite programming $\cdot$ Delsarte

Mathematics Subject Classification 94B65 - 05E10 - 90C22 · 20C30

\section{Introduction}

Let $\mathbb{Z}_{+}$denote the set of nonnegative integers, and denote $[m]=\{1, \ldots, m\}$, for any $m \in \mathbb{Z}_{+}$. Fixing $n, q \in \mathbb{Z}_{+}$, a code is a subset of $[q]^{n}$. So $[q]$ serves as the alphabet and $n$ as the word length. We will assume throughout that $q \geq 2$. (If you prefer $\{0,1, \ldots, q-1\}$ as alphabet, take the letters $\bmod q$.) While this paper is mainly meant to handle the case $q \geq 3$, the results also hold for $q=2$.

For $v, w \in[q]^{n}$, the (Hamming) distance $d_{H}(v, w)$ is equal to the number of $i \in[n]$ with $v_{i} \neq w_{i}$. The minimum distance of a code $C$ is the minimum of $d_{H}(v, w)$ taken over distinct

This is one of several papers published in Designs, Codes and Cryptography comprising the special issue in honor of Andries Brouwer's 65th birthday.

Alexander Schrijver

lex@cwi.nl

1 Korteweg-De Vries Institute for Mathematics, University of Amsterdam, Amsterdam, The Netherlands 
$v, w \in C$. Then $A_{q}(n, d)$ denotes the maximum cardinality of a code with minimum distance at least $d$. We will study the following upper bound on $A_{q}(n, d)$, sharpening Delsarte's classical linear programming bound [4].

For $k \in \mathbb{Z}_{+}$, let $\mathcal{C}_{k}$ be the collection of subsets $C$ of $[q]^{n}$ with $|C| \leq k$. For each $x: \mathcal{C}_{4} \rightarrow \mathbb{R}$ define the $\mathcal{C}_{2} \times \mathcal{C}_{2}$ matrix $M(x)$ by

$$
M(x)_{C, C^{\prime}}:=x\left(C \cup C^{\prime}\right)
$$

for $C, C^{\prime} \in \mathcal{C}_{2}$. Then define

$$
B_{q}(n, d):=\max _{x} \sum_{w \in[q]^{n}} x(\{w\}), \text { where } x: \mathcal{C}_{4} \rightarrow \mathbb{R}_{+} \text {satisfies }
$$

(i) $x(\emptyset)=1$,

(ii) $x(C)=0$ if the minimum distance of $C$ is less than $d$,

(iii) $M(x)$ is positive semidefinite.

Proposition $1 A_{q}(n, d) \leq B_{q}(n, d)$.

Proof Let $D \subseteq[q]^{n}$ have minimum distance at least $d$ and satisfy $|D|=A_{q}(n, d)$. Define $x: \mathcal{C}_{4} \rightarrow \mathbb{R}$ by $x(C)=1$ if $C \subseteq D$ and $x(C)=0$ otherwise. Then $x$ satisfies the conditions: (iii) follows from the fact that for this $x$ one has $M(x)_{C, C^{\prime}}=x(C) x\left(C^{\prime}\right)$ for all $C, C^{\prime} \in \mathcal{C}_{2}$. Moreover, $\sum_{w \in[q]^{n}} x(\{w\})=|D|=A_{q}(n, d)$.

The optimization problem (2) is huge, but, with methods from representation theory, can be reduced to a size bounded by a polynomial in $n$, with entries (i.e., coefficients) being polynomials in $q$. This makes it possible to solve (2) by semidefinite programming for some moderate values of $n, d$, and $q$, leading to improvements of best known upper bounds for $A_{q}(n, d)$.

To explain the reduction, let $H$ be the wreath product $S_{q}^{n} \rtimes S_{n}$. For each $k$, the group $H$ acts naturally on $\mathcal{C}_{k}$, maintaining minimum distances and cardinalities of elements of $\mathcal{C}_{k}$ (being codes). Then we can assume that $x$ is invariant under the $H$-action on $\mathcal{C}_{4}$. That is, we can assume that $x(C)=x(D)$ whenever $C, D \in \mathcal{C}_{2}$ and $D=g \cdot C$ for some $g \in H$. Indeed, (2)(i)(ii)(iii) are maintained under replacing $x$ by $g \cdot x$. (Note that $M(g \cdot x)$ is obtained from $M(x)$ by simultaneously permuting rows and columns.) Moreover, the objective function does not change by this action. Hence the optimum $x$ can be replaced by the average of all $g \cdot x$ (over all $g \in H$ ), by the convexity of the set of positive semidefinite matrices. This makes the optimum solution $H$-invariant.

Let $\Omega$ be the set of $H$-orbits on $\mathcal{C}_{4}$. Note that $\Omega$ is bounded by a polynomial in $n$ (independently of $q$ ). As there exists an $H$-invariant optimum solution, we can replace, for each $\omega \in \Omega$ and $C \in \omega$, each variable $x(C)$ by a variable $y(\omega)$. In this way we obtain $M(y)$.

Then $M(y)$ is invariant under the action of $H$ on its rows and columns, induced from the action of $H$ on $\mathcal{C}_{2}$. Hence $M(y)$ can be block-diagonalized by $M(y) \mapsto U^{\top} M(y) U$, where $U$ is a matrix independent of $y$. The entries in each block are linear functions of the variables $y(\omega)$. There are several equal (or equivalent) blocks. Taking one block from each such class gives a matrix of order polynomial in $n$ with numbers that are polynomials in $q$. The issue crucial for us is that the original matrix $M(y)$ is positive semidefinite if and only if each of the blocks is positive semidefinite.

In this paper we will describe the blocks that reduce the problem. With this, we found the following improvements on the known bounds for $A_{q}(n, d)$, with thanks to Hans D. Mittelmann for his help in solving the larger-sized problems. 


\begin{tabular}{llllll}
\hline$q$ & $n$ & $d$ & Best lower bound known & New upper bound & Best upper bound previously known \\
\hline 4 & 6 & 3 & 164 & 176 & 179 \\
4 & 7 & 3 & 512 & 596 & 614 \\
4 & 7 & 4 & 128 & 155 & 169 \\
5 & 7 & 4 & 250 & 489 & 545 \\
5 & 7 & 5 & 53 & 87 & 108 \\
\hline
\end{tabular}

The best upper bounds previously known for $A_{4}(6,3)$ and $A_{4}(7,3)$ are Delsarte's linear programming bound [4]; the other three best upper bounds previously known were given by Gijswijt, Schrijver, and Tanaka [7]. We refer to the most invaluable tables maintained by Andries Brouwer [3] with the best known lower and upper bounds for the size of errorcorrecting codes (see also Bogdanova, Brouwer, Kapralov, and Östergård [1] and Bogdanova and Östergård [2] for studies of bounds for codes over alphabets of size $q=4$ and $q=5$, respectively).

\subsection{Comparison with earlier bounds}

The bound $B_{q}(n, d)$ described above is a sharpening of Delsarte's classical linear programming bound [4]. The value of the Delsarte bound is equal to our bound after replacing $\mathcal{C}_{4}$ and $\mathcal{C}_{2}$ by $\mathcal{C}_{2}$ and $\mathcal{C}_{1}$, respectively, which generally yields a less strict bound.

We can add to (2) the condition that, for each $D \in \mathcal{C}_{4}$, the $S(D) \times S(D)$ matrix

$$
\left(x\left(C \cup C^{\prime}\right)\right)_{C, C^{\prime} \in S(D)} \text { is positive semidefinite, }
$$

where $S(D):=\left\{C \in \mathcal{C}_{4}|C \supseteq D| D,|+2| C \backslash D \mid \leq 4\right\}$. (So (iii) in (2) is the case $D=\emptyset$.) Also the addition of (3) allows a reduction of the optimization problem to polynomial size as above. (It can be seen that adding (3) for $|D|=2$ suffices.) For $q=2$ we obtain in this way the bound given by Gijswijt et al. [6]. Our present description gives a more conceptual and representation-theoretic approach to the method of [6].

A bound intermediate to the Delsarte bound and the currently investigated bound is based on considering functions $x: \mathcal{C}_{3} \rightarrow \mathbb{R}_{+}$and the related matrices-see Schrijver [9] for binary codes and Gijswijt et al. [7] for nonbinary codes.

\section{Preliminaries on representation theory}

We assume some familiarity with classical representation theory, in particular of the symmetric group $S_{n}$ and of finite groups in general. In this section we give a brief review, also to settle some notation and terminology. We refer to Sagan [8] for background.

A group $G$ acts on a set $X$ if there is a group homomorphism $G \rightarrow S_{X}$, where $S_{X}$ is the group of bijections $X \rightarrow X$. The image of $g \in G$ in $S_{X}$ is indicated by $g \cdot$. If $X$ is a linear space, the bijections are assumed to be linear functions. The action of $G$ on a set $X$ induces an action of $G$ on the linear space $\mathbb{C}^{X}$, by $(g \cdot f)(x):=f\left(g^{-1} \cdot x\right)$ for all $g \in G, f \in \mathbb{C}^{X}$, and $x \in X$. If any group $G$ acts on $X$, then $X^{G}$ denotes the set of elements of $X$ invariant under the action of $G$.

Let $m \in \mathbb{Z}_{+}$and let $G$ be a finite group acting unitarily on $V=\mathbb{C}^{m}$ (meaning that for each $g \in G$ there is a unitary $m \times m$ matrix $U$ such that $g \cdot x=U x$ for all $x \in \mathbb{C}^{m}$ ). Then $V$ can be decomposed uniquely as direct sum of the $G$-isotypical components $V_{1}, \ldots, V_{k}$. 
For distinct $i, j, V_{i}$ and $V_{j}$ are orthogonal (with respect to the inner product $\langle x, y\rangle=x^{*} y$ for $x, y \in \mathbb{C}^{m}$, where $x^{*}$ is the conjugate transpose of $x$ ). Next, each $V_{i}$ is a direct sum $V_{i, 1} \oplus \cdots \oplus V_{i, m_{i}}$ of mutually $G$-isomorphic, irreducible $G$-modules, in such a way that $V_{i, j}$ and $V_{i, j^{\prime}}$ are orthogonal for distinct $j, j^{\prime}$. (This decomposition is generally not unique.) For each $i \leq k$ and $j \leq m_{i}$, choose a nonzero $u_{i, j} \in V_{i, j}$ such that for each $i$ and all $j, j^{\prime} \leq m_{i}$ there exists a $G$-isomorphism $V_{i, j} \rightarrow V_{i, j^{\prime}}$ bringing $u_{i, j}$ to $u_{i, j^{\prime}}$. For each $i \leq k$, let $U_{i}$ be the matrix $\left[u_{i, 1}, \ldots, u_{i, m_{i}}\right]$, considering the $u_{i, j}$ as columns. We call any matrix set $\left\{U_{1}, \ldots, U_{k}\right\}$ that can be obtained in this way representative for the action of $G$ on $\mathbb{C}^{m}$. It has the property that the function

$$
\Phi:\left(\mathbb{C}^{m \times m}\right)^{G} \rightarrow \bigoplus_{i=1}^{k} \mathbb{C}^{m_{i} \times m_{i}} \quad \text { with } \quad \Phi(X):=\bigoplus_{i=1}^{k} U_{i}^{*} X U_{i}
$$

for $X \in\left(\mathbb{C}^{m \times m}\right)^{G}$ is bijective. So $\sum_{i} m_{i}^{2}$ is equal to the dimension of $\left(\mathbb{C}^{m \times m}\right)^{G}$ (and hence can be considerably smaller than $m$ ).

Another important property of a representative matrix set is that any $X \in\left(\mathbb{C}^{m \times m}\right)^{G}$ is positive semidefinite if and only if $\Phi(X)$ is positive semidefinite. (A positive semidefinite matrix is a Hermitian matrix with all eigenvalues nonnegative.)

In our applications below, throughout $G$ is acting real-orthogonally on a vector space $V=\mathbb{R}^{m}$; that is, for each $g \in G$ there is a real orthogonal $m \times m$ matrix $U$ with $g \cdot x=U x$ for each $x \in \mathbb{C}^{m}$.

Moreover, as it turns out, for the cases considered in the present paper the matrices $U_{i}$ can be taken real-valued (which is computationally convenient). This implies that $\Phi(X)=$ $\bigoplus_{i=1}^{k} U_{i}^{\top} X U_{i}$ for $X \in\left(\mathbb{R}^{m \times m}\right)^{G}$ and $\Phi\left(\left(\mathbb{R}^{m \times m}\right)^{G}\right)=\bigoplus_{i=1}^{k} \mathbb{R}^{m_{i} \otimes m_{i}}$. Moreover, a matrix $X \in \mathbb{R}^{m \times m}$ is positive semidefinite if and only if $U_{i}^{\top} X U_{i}$ is positive semidefinite for each $i=1, \ldots, k$. For later reference we state that, since for all $i, j, V_{i, j}$ is the linear space spanned by $G \cdot u_{i, j}$,

$$
\mathbb{R}^{m}=\bigoplus_{i=1}^{k} \bigoplus_{j=1}^{m_{i}} \mathbb{R} G \cdot u_{i, j}
$$

It will turn out to be convenient to consider the columns of the matrices $U_{i}$ as elements of the dual space $\left(\mathbb{R}^{m}\right)^{*}$ (by taking the standard inner product). Then each $U_{i}$ is an ordered set of linear functions on $\mathbb{R}^{m}$. (The order plays a role in describing a representative matrix set for the action of the wreath product $G^{n} \rtimes S_{n}$ on $V^{\otimes n}$.)

\subsection{A representative set for the action of $S_{n}$ on $V^{\otimes n}$}

Classical representation theory of the symmetric group yields a representative set for the natural action of $S_{n}$ on $V^{\otimes n}$, where $V$ is a finite-dimensional vector space, which we will describe now.

For $n \in \mathbb{Z}_{+}, \lambda \vdash n$ means that $\lambda$ is equal to $\left(\lambda_{1}, \ldots, \lambda_{t}\right)$ for some $t$, with $\lambda_{1} \geq \cdots \geq \lambda_{t}>0$ integer and $\lambda_{1}+\cdots+\lambda_{t}=n$. The number $t$ is called the height of $\lambda$. The Young shape $Y(\lambda)$ of $\lambda$ is the set

$$
Y(\lambda):=\left\{(i, j) \in \mathbb{Z}_{+}^{2} \mid 1 \leq j \leq t, 1 \leq i \leq \lambda_{j}\right\} .
$$

For any $j_{0} \leq t$, the set of elements $\left(i, j_{0}\right)$ in $Y(\lambda)$ is called the $j_{0}$-th row of $Y(\lambda)$. Let $R_{\lambda}$ be the group of permutations $\pi$ of $Y(\lambda)$ with $\pi(Z)=Z$ for each row $Z$ of $Y(\lambda)$. For any 
$i_{0} \leq \lambda_{1}$, the set of elements $\left(i_{0}, j\right)$ in $Y(\lambda)$ is called the $i_{0}$-th column of $Y(\lambda)$. Let $C_{\lambda}$ be the group of permutations $\pi$ of $Y(\lambda)$ with $\pi(Z)=Z$ for each column $Z$ of $Y(\lambda)$.

A $\lambda$-tableau is a function $\tau: Y(\lambda) \rightarrow \mathbb{Z}_{+}$. We put $\tau \sim \tau^{\prime}$ for $\lambda$-tableaux $\tau, \tau^{\prime}$ if $\tau^{\prime}=\tau r$ for some $r \in R_{\lambda}$. A $\lambda$-tableau is semistandard if in each row the entries are nondecreasing and in each column the entries are increasing. Let $T_{\lambda, m}$ denote the collection of semistandard $\lambda$-tableaux with entries in $[m]$. Note that $T_{\lambda, m} \neq \emptyset$ if and only if $\lambda$ has height at most $m$.

Let $B=(B(1), \ldots, B(m))$ be an ordered basis of $V^{*}$. For $\tau \in T_{\lambda, m}$, define the following element of $\left(V^{*}\right)^{\otimes n}$ :

$$
u_{\tau, B}:=\sum_{\tau^{\prime} \sim \tau} \sum_{c \in C_{\lambda}} \operatorname{sgn}(c) \bigotimes_{y \in Y(\lambda)} B\left(\tau^{\prime} c(y)\right),
$$

where we order the Young shape $Y(\lambda)$ by concatenating its rows. Then the matrix set

$$
\left\{\left[u_{\tau, B} \mid \tau \in T_{\lambda, m}\right] \mid \lambda \vdash n\right\}
$$

is representative for the natural action of $S_{n}$ on $V^{\otimes n}$.

\section{Reduction of the optimization problem}

In this section we describe reducing the optimization problem (2) conceptually. In Sect. 4 we consider the reduction computationally. For the remainder of this paper we fix $n$ and $q$.

We consider the natural action of $H=S_{q}^{n} \rtimes S_{n}$ on $\mathbb{R}^{\mathcal{C}_{2}}$. If $U_{1}, \ldots, U_{k}$ form a representative set of matrices for this action, then with (4) we obtain a reduction of the size of the optimization problem to polynomial size. To make this reduction explicit in order to apply semidefinite programming, we need to express each $m_{i} \times m_{i}$ matrix $U_{i}^{\top} M(y) U_{i}$ as an explicit matrix in which each entry is a linear combination of the variables $y(\omega)$ for $\omega \in \Omega$ (the set of $H$-orbits of $\mathcal{C}_{4}$ ).

For $\omega \in \Omega$, let $N_{\omega}$ be the $\mathcal{C}_{2} \times \mathcal{C}_{2}$ matrix with 0,1 entries satisfying

$$
\left(N_{\omega}\right)_{\{\alpha, \beta\},\{\gamma, \delta\}}=1 \text { if and only if }\{\alpha, \beta, \gamma, \delta\} \in \omega
$$

for $\alpha, \beta, \gamma, \delta \in[q]^{n}$. Then

$$
U_{i}^{\top} M(y) U_{i}=\sum_{\omega} y(\omega) U_{i}^{\top} N_{\omega} U_{i}
$$

So to get the reduction, we need to obtain the matrices $U_{i}^{\top} N_{\omega} U_{i}$ explicitly, for each $\omega \in \Omega$ and for each $i=1, \ldots, k$. We do this in a number of steps.

We first describe in Sect. 3.1 a representative set for the natural action of $S_{q}$ on $\mathbb{R}^{q \times q}$. From this we derive, in Sect. 3.2, with the help of the representative set for the action of $S_{n}$ on $V^{\otimes n}$ described in Sect. 2.1, a representative set for the action of the wreath product $H=S_{q}^{n} \rtimes S_{n}$ on the set $\left([q]^{n}\right)^{2}$ of ordered pairs of words in $[q]^{n}$, in other words, on $\mathbb{R}^{\left([q]^{n}\right)^{2}} \cong\left(\mathbb{R}^{q \times q}\right)^{\otimes n}$. From this we derive in Sect. 3.3 a representative set for the action of $H$ on the set $\mathcal{C}_{2} \backslash\{\emptyset\}$ of unordered pairs $\{v, w\}$ (including singleton) of words $v, w$ in $[q]^{n}$. Then in Sect. 3.4 we derive a representative set for the action of $H$ on the $\operatorname{set} \mathcal{C}_{2}^{d} \backslash\{\emptyset\}$, where $\mathcal{C}_{2}^{d}$ is the set of codes in $\mathcal{C}_{2}$ of minimum distance at least $d$. (So each singleton word belongs to $\mathcal{C}_{2}^{d}$.) Finally, in Sect. 3.4 we include the empty set $\emptyset$, by an easy representation-theoretic argument. 


\subsection{A representative set for the action of $S_{q}$ on $\mathbb{R}^{q \times q}$}

We now consider the natural action of $S_{q}$ on $\mathbb{R}^{q \times q}$. Let $e_{j}$ be the $j$-th unit basis vector in $\mathbb{R}^{q}$, $I_{q}$ be the $q \times q$ identity matrix, $J_{q}$ be the all-one $q \times q$ matrix, 1 be the all-one column vector in $\mathbb{R}^{q}, N:=\left(e_{1}-e_{2}\right) \mathbf{1}^{\top}$, and $E_{i, j}:=e_{i} e_{j}^{\top}$. We furthermore define the following matrices, where we consider matrices in $\mathbb{R}^{q \times q}$ as columns of the matrices $B_{i}$ :

$$
\begin{aligned}
& B_{1}:=\left[I_{q}, J_{q}-I_{q}\right], \\
& B_{2}:=\left[E_{1,1}-E_{2,2}, N-N^{\top}, N+N^{\top}-2\left(E_{1,1}-E_{2,2}\right)\right], \\
& B_{3}:=\left[E_{1,2}+E_{2,3}+E_{3,1}-E_{2,1}-E_{3,2}-E_{1,3}\right], \\
& B_{4}:=\left[E_{1,3}-E_{3,2}+E_{2,4}-E_{4,1}+E_{3,1}-E_{2,3}+E_{4,2}-E_{1,4}\right] .
\end{aligned}
$$

The matrices in $\mathbb{R}^{q \times q}$ will in fact be taken as elements of the dual space ( $\left.\mathbb{R}^{q \times q}\right)^{*}$ (by taking the inner product), so that they are elements of the algebra $\mathcal{O}\left(\mathbb{R}^{q \times q}\right)$ of polynomials on the linear space $\mathbb{R}^{q \times q}$.

Then $\left\{B_{1}, \ldots, B_{4}\right\}$ is representative for the natural action of $S_{q}$ on $\mathbb{R}^{q \times q}$, if $q \geq 4$. If $q \leq 3$, we delete $B_{4}$, and if $q=2$ we moreover delete $B_{3}$ and the last column of $B_{2}$ (as this column is 0 if $q=2$ ). We give a proof in Appendix 1 .

If $q \geq 4$, set $k=4, m_{1}=2, m_{2}:=3, m_{3}:=1$, and $m_{4}:=1$. If $q=3$, set $k=3, m_{1}=2$, $m_{2}:=3$, and $m_{3}:=1$. If $q=2$, set $k=2, m_{1}=2$, and $m_{2}:=2$. For the remainder of this paper we fix $k, m_{1}, \ldots, m_{k}$, and $B_{1}, \ldots, B_{k}$.

\subsection{A representative set for the action of $H$ on $\left(\mathbb{R}^{q \times q}\right)^{\otimes n}$}

Recall that $H=S_{q}^{n} \rtimes S_{n}$ and that we have fixed $k, m_{1}, \ldots, m_{k}$, and $B_{1}, \ldots, B_{k}$ in Sect. 3.1.

We next consider the action of $H$ on the set $\left([q]^{n}\right)^{2}$ of ordered pairs of code words. For that, we derive a representative set for the natural action of $H$ on $\left(\mathbb{R}^{q \times q}\right)^{\otimes n} \cong \mathbb{R}^{\left([q]^{n}\right)^{2}}$ from the results described in Sects. 2.1 and 3.1.

Let $N$ be the collection of all $k$-tuples $\left(n_{1}, \ldots, n_{k}\right)$ of nonnegative integers adding up to $n$. For $\boldsymbol{n}=\left(n_{1}, \ldots, n_{k}\right) \in \boldsymbol{N}$, let $\lambda \vdash \boldsymbol{n}$ mean that $\lambda=\left(\lambda_{1}, \ldots, \lambda_{k}\right)$ with $\lambda_{i} \vdash n_{i}$ for $i=1, \ldots, k$. (So each $\lambda_{i}$ is equal to $\left(\lambda_{i, 1}, \ldots, \lambda_{i, t}\right)$ for some $t$.)

For $\lambda \vdash \boldsymbol{n}$ define

$$
W_{\lambda}:=T_{\lambda_{1}, m_{1}} \times \cdots \times T_{\lambda_{k}, m_{k}}
$$

and for $\boldsymbol{\tau}=\left(\tau_{1}, \ldots, \tau_{k}\right) \in W_{\lambda}$ define

$$
v_{\boldsymbol{\tau}}:=\bigotimes_{i=1}^{k} u_{\tau_{i}, B_{i}}
$$

Proposition 2 The matrix set

$$
\left\{\left[v_{\boldsymbol{\tau}} \mid \boldsymbol{\tau} \in W_{\lambda}\right] \quad \mid \boldsymbol{n} \in \boldsymbol{N}, \lambda \vdash \boldsymbol{n}\right\}
$$

is representative for the action of $S_{q}^{n} \rtimes S_{n}$ on $\left(\mathbb{R}^{q \times q}\right)^{\otimes n}$. 
Proof Let $L_{i}$ denote the linear space spanned by $B_{i}(1), \ldots, B_{i}\left(m_{i}\right)$. Then

$$
\begin{gathered}
\left(\mathbb{R}^{q \times q}\right)^{\otimes n} \stackrel{\text { by (5) }}{=}\left(\bigoplus_{i=1}^{k} \bigoplus_{j=1}^{m_{i}} \mathbb{R} S_{q} \cdot B_{i}(j)\right)^{\otimes n}=\mathbb{R} S_{n} \cdot \bigoplus_{n \in N} \bigoplus_{i=1}^{k}\left(\bigoplus_{j=1}^{m_{i}} \mathbb{R} S_{q} \cdot B_{i}(j)\right)^{\otimes n_{i}} \\
=\mathbb{R} S_{n} \cdot \mathbb{R} S_{q}^{\otimes n} \cdot \bigoplus_{n \in N} \bigotimes_{i=1}^{k} L_{i}^{\otimes n_{i}} \stackrel{\text { by (5) }}{=} H \cdot \bigoplus_{n \in N}^{k} \bigoplus_{i=1} \bigoplus_{\lambda_{i} \vdash n_{i}} \mathbb{R}_{i} S_{\lambda_{\lambda_{i}}, m_{i}} \cdot u_{\tau_{i}, B_{i}} \\
=\bigoplus_{\boldsymbol{n} \in N} \bigoplus_{\lambda \vdash \boldsymbol{n}} \bigoplus_{\boldsymbol{\tau} \in W_{\lambda}} \mathbb{R} H \cdot v_{\boldsymbol{\tau}} .
\end{gathered}
$$

Now for each $\boldsymbol{n}, \lambda$ and $\boldsymbol{\tau}, \boldsymbol{\sigma} \in W_{\boldsymbol{\lambda}}$, there is an $H$-isomorphism $\mathbb{R} H \cdot v_{\boldsymbol{\tau}} \rightarrow \mathbb{R} H \cdot v_{\boldsymbol{\sigma}}$ bringing $v_{\tau}$ to $v_{\sigma}$, since for each $i=1, \ldots, k$, setting $H_{i}:=S_{q}^{n_{i}} \rtimes S_{n_{i}}$, there is an $H_{i}$-isomorphism $\mathbb{R} H_{i} \cdot u_{\tau_{i}, B_{i}} \rightarrow \mathbb{R} H_{i} \cdot u_{\sigma_{i}, B_{i}}$. Hence $\left(\right.$ where $\operatorname{Sym}_{t}(X):=\left(X^{\otimes t}\right)^{S_{t}}$ for any $t \in \mathbb{Z}_{+}$and linear space $X$, with the natural action of $S_{t}$ on $X^{\otimes t}$ )

$$
\begin{aligned}
& \operatorname{dim}\left(\left(\mathbb{R}^{q \times q}\right)^{\otimes n} \otimes\left(\mathbb{R}^{q \times q}\right)^{\otimes n}\right)^{H} \geq \sum_{\boldsymbol{n} \in \boldsymbol{N}} \sum_{\lambda \vdash \boldsymbol{n}}\left|W_{\lambda}\right|^{2}=\sum_{\boldsymbol{n} \in \boldsymbol{N}} \sum_{\lambda \vdash \boldsymbol{n}} \prod_{i=1}^{k}\left|T_{\lambda_{i}, m_{i}}\right|^{2} \\
& =\sum_{n \in N} \prod_{i=1}^{k} \sum_{\lambda_{i} \vdash n_{i}}\left|T_{\lambda_{i}, m_{i}}\right|^{2} \\
& \stackrel{\text { by (8) }}{=} \sum_{n \in N} \prod_{i=1}^{k} \operatorname{dim} \operatorname{Sym}_{n_{i}}\left(\mathbb{R}^{m_{i}} \otimes \mathbb{R}^{m_{i}}\right) \\
& =\sum_{n \in N} \prod_{i=1}^{k}\left(\begin{array}{c}
m_{i}^{2}+n_{i}-1 \\
n_{i}-1
\end{array}\right)=\left(\begin{array}{c}
\sum_{i=1}^{k} m_{i}^{2}+n-1 \\
n-1
\end{array}\right) \\
& =\operatorname{dim} \operatorname{Sym}_{n}\left(\left(\left(\mathbb{R}^{q \times q}\right) \otimes\left(\mathbb{R}^{q \times q}\right)\right)^{S_{q}}\right) \\
& =\operatorname{dim}\left(\left(\mathbb{R}^{q \times q}\right)^{\otimes n} \otimes\left(\mathbb{R}^{q \times q}\right)^{\otimes n}\right)^{H}
\end{aligned}
$$

as $\sum_{i=1}^{k} m_{i}^{2}=\operatorname{dim}\left(\mathbb{R}^{q \times q} \otimes \mathbb{R}^{q \times q}\right)^{S_{q}}$. So we have equality throughout in (16), and hence each $\mathbb{R} H \cdot v_{\tau}$ is irreducible, and if $\lambda \neq \lambda^{\prime}$, then for each $\tau \in W_{\lambda}$ and $\tau^{\prime} \in W_{\lambda^{\prime}}, \mathbb{R} H \cdot v_{\tau}$ and $\mathbb{R} H \cdot v_{\tau^{\prime}}$ are not $H$-isomorphic.

\subsection{Unordered pairs}

We now go over from the set $\left([q]^{n}\right)^{2}$ of ordered pairs of code words to the set $\mathcal{C}_{2} \backslash\{\emptyset\}$ of unordered pairs (including singletons) of code words. For this we consider the action of the group $S_{2}$ on $\mathbb{R}^{[q]^{n} \times[q]^{n}} \cong \mathbb{R}^{\left([q]^{n}\right)^{2}} \cong\left(\mathbb{R}^{q \times q}\right)^{\otimes n}$, where the nonidentity element $\sigma$ in $S_{2}$ acts as taking the transpose. The actions of $S_{2}$ and $H$ commute.

Let $F$ be the $\left(\mathcal{C}_{2} \backslash\{\emptyset\}\right) \times\left([q]^{n}\right)^{2}$ matrix with 0,1 entries satisfying

$$
F_{\{\alpha, \beta\},(\gamma, \delta)}=1 \text { if and only if }\{\gamma, \delta\}=\{\alpha, \beta\},
$$

for $\alpha, \beta, \gamma, \delta \in[q]^{n}$. Then the function $x \mapsto F x$ is an $H$-isomorphism $\left(\mathbb{R}^{\left([q]^{n}\right)^{2}}\right)^{S_{2}} \rightarrow$ $\mathbb{R}^{\mathcal{C}_{2} \backslash\{\emptyset\}}$.

Now note that each $B_{i}(j)$, as matrix in $\mathbb{R}^{q \times q}$, is $S_{2}$-invariant (i.e., symmetric) except for $B_{2}(2)$ and $B_{3}(1)$, while $\sigma \cdot B_{2}(2)=-B_{2}(2)$ and $\sigma \cdot B_{3}(1)=-B_{3}(1)$ (as $B_{2}(2)$ and $B_{3}(1)$ are skew-symmetric). So for any $\boldsymbol{n} \in \boldsymbol{N}, \boldsymbol{\lambda} \vdash \boldsymbol{n}$, and $\boldsymbol{\tau} \in W_{\lambda}$, we have 


$$
\sigma \cdot v_{\tau}=(-1)^{\left|\tau_{2}^{-1}(2)\right|+\left|\tau_{3}^{-1}(1)\right|} v_{\tau}
$$

Therefore, let $W_{\lambda}^{\prime}$ be the set of those $\tau \in W_{\lambda}$ with $\left|\tau_{2}^{-1}(2)\right|+\left|\tau_{3}^{-1}(1)\right|$ even. Then the matrix set

$$
\left\{\left[v_{\boldsymbol{\tau}} \mid \boldsymbol{\tau} \in W_{\lambda}^{\prime}\right] \quad \mid \boldsymbol{n} \in \boldsymbol{N}, \boldsymbol{\lambda} \vdash \boldsymbol{n}\right\}
$$

is representative for the action of $H$ on $\left(\mathbb{R}^{\left([q]^{n}\right)^{2}}\right)^{S_{2}}$. Hence the matrix set

$$
\left\{\left[F v_{\tau} \mid \boldsymbol{\tau} \in W_{\lambda}^{\prime}\right] \mid \boldsymbol{n} \in \boldsymbol{N}, \boldsymbol{\lambda} \vdash \boldsymbol{n}\right\}
$$

is representative for the action of $H$ on $\mathbb{R}^{\mathcal{C}_{2} \backslash\{\emptyset\}}$.

\subsection{Restriction to pairs of words at distance at least $d$}

Let $d \in \mathbb{Z}_{+}$, and let $\mathcal{C}_{2}^{d}$ be the collection of elements of $\mathcal{C}_{2}$ of minimum distance at least $d$. Note that each singleton code word belongs to $\mathcal{C}_{2}^{d}$, and that $H$ acts on $\mathcal{C}_{2}^{d}$. From (20) we derive a representative set for the action of $H$ on $\mathbb{R}_{2}^{\mathcal{C}_{2}^{d} \backslash\{\emptyset\}}$.

To see this, let for each $t \in \mathbb{Z}_{+}, L_{t}$ be the subspace of $\mathbb{R}^{\mathcal{C}_{2}}$ spanned by the elements $e_{\{\alpha, \beta\}}$ with $\alpha, \beta \in[q]^{n}$ and $d_{H}(\alpha, \beta)=t$. (For any $Z \in \mathcal{C}_{2}, e_{Z}$ denotes the unit base vector in $\mathbb{R}^{\mathcal{C}_{2}^{d}}$ for coordinate $Z$.)

Then for any $\boldsymbol{n} \in \boldsymbol{N}, \lambda \vdash \boldsymbol{n}$, and $\boldsymbol{\tau} \in W_{\lambda}^{\prime}$, the irreducible representation $H \cdot F v_{\boldsymbol{\tau}}$ is contained in $L_{t}$, where

$$
t:=n-\left|\tau_{1}^{-1}(1)\right|-\left|\tau_{2}^{-1}(1)\right|,
$$

since $B_{1}(1)=I_{q}$ and $B_{2}(1)=E_{1,1}-E_{2,2}$ are the only two entries $B_{i}(j)$ in the $B_{i}$ that have nonzeros on the diagonal of the matrix $B_{i}(j)$. Let $W_{\lambda}^{\prime \prime}$ be the set of those $\tau$ in $W_{\lambda}^{\prime}$ with

$$
n-\left|\tau_{1}^{-1}(1)\right|-\left|\tau_{2}^{-1}(1)\right| \in\{0, d, d+1, \ldots, n\} .
$$

Then a representative set for the action of $H$ on $\mathcal{C}_{2}^{d} \backslash\{\emptyset\}$ is

$$
\left\{\left[F v_{\tau} \mid \boldsymbol{\tau} \in W_{\lambda}^{\prime \prime}\right] \mid \boldsymbol{n} \in \boldsymbol{N}, \lambda \vdash \boldsymbol{n}\right\} .
$$

\subsection{Adding $\emptyset$}

To obtain a representative set for the action of $H$ on $\mathcal{C}_{2}^{d}$, note that $H$ acts trivially on $\emptyset$. So $e_{\emptyset}$ belongs to the $H$-isotypical component of $\mathbb{R}^{\mathcal{C}_{2}}$ that consists of $H$-invariant elements. Now the $H$-isotypical component of $\mathbb{R}^{\mathcal{C}_{2} \backslash\{\emptyset\}}$ that consists of the $H$-invariant elements corresponds to the matrix in the representative set indexed by indexed by $\boldsymbol{n}=(n, 0,0,0)$ and $\boldsymbol{\lambda}=$ $((n),(),(),())$, where ()$\vdash 0$. So to obtain a representative set for $\mathbb{R}^{\mathcal{C}_{2}}$, we just add $e_{\emptyset}$ as column to this matrix.

\section{How to compute $\left(F v_{\tau}\right)^{\top} N_{\omega} F v_{\sigma}$}

We now have a reduction of the original problem to blocks with coefficients $\left(F v_{\tau}\right)^{\top} N_{\omega} F v_{\sigma}$, for $\boldsymbol{n} \in \boldsymbol{N}, \boldsymbol{\lambda} \vdash \boldsymbol{n}, \boldsymbol{\tau}, \boldsymbol{\sigma} \in W_{\lambda}$, and $\omega \in \Omega$. The number and orders of these blocks are bounded by a polynomial in $n$, but computing these coefficients still must be reduced in time, since the order of $F, v_{\tau}, v_{\sigma}$, and $N_{\omega}$ is exponential in $n$. 
Fix $\boldsymbol{n} \in \boldsymbol{N}, \boldsymbol{\lambda} \vdash \boldsymbol{n}$, and $\boldsymbol{\tau}, \boldsymbol{\sigma} \in W_{\lambda}$. For any $\omega \in \Omega$, let $L_{\omega}:=F^{\top} N_{\omega} F$. So $L_{\omega}$ is a $\left([q]^{n} \times[q]^{n}\right) \times\left([q]^{n} \times[q]^{n}\right)$ matrix with 0,1 entries satisfying

$$
\left(L_{\omega}\right)_{(\alpha, \beta),(\gamma, \delta)}=1 \text { if and only if }\{\alpha, \beta, \gamma, \delta\} \in \omega,
$$

for all $\alpha, \beta, \gamma, \delta \in[q]^{n}$. By definition of $L_{\omega}$,

$$
\left(F v_{\tau}\right)^{\top} N_{\omega} F v_{\sigma}=v_{\tau}^{\top} L_{\omega} v_{\sigma}
$$

So it suffices to evaluate the latter value.

Let $\Pi$ be the collection of partitions of $\{1,2,3,4\}$ into at most $q$ parts. There is the following bijection between $\Pi$ and the set of orbits of the action of $S_{q}$ on $[q]^{4}$.

For each word $w \in[q]^{4}$, let $\operatorname{part}(w)$ be the partition $P \in \Pi$ such that $i$ and $j$ belong to the same class of $P$ if and only if $w_{i}=w_{j}$ (for $i, j=1, \ldots, 4$ ). Then two elements $v, w \in[q]^{4}$ belong to the same $S_{q}$-orbit if and only if $\operatorname{part}(v)=\operatorname{part}(w)$. Note that $|\Pi|=8$ if $q=2$, $|\Pi|=14$ if $q=3$, and $|\Pi|=15$ if $q \geq 4$. (In all cases, $|\Pi|=\operatorname{dim}\left(\mathbb{R}^{q \times q}\right)^{S_{q}}=\sum_{i=1}^{k} m_{i}^{2}$.)

For $P \in \Pi$, let

$$
d_{P}:=\sum_{\substack{i_{1}, \ldots, i_{4} \in[q] \\ \text { part } i_{1} \cdots i_{4}=P}} e_{i_{1}} e_{i_{2}}^{\top} \otimes e_{i_{3}} e_{i_{4}}^{\top}
$$

where each $e_{i}$ is a unit basis column vector in $\mathbb{R}^{q}$, so that $e_{i} e_{j}^{\top}$ is a matrix in $\mathbb{R}^{q \times q}$. Then $D:=\left\{d_{P} \mid P \in \Pi\right\}$ is a basis of $\left(\mathbb{R}^{q \times q} \otimes \mathbb{R}^{q \times q}\right)^{S_{q}}$. Let $D^{*}$ be the dual basis.

For any $(\alpha, \beta, \gamma, \delta) \in\left([q]^{n}\right)^{4}$, let

$$
\psi(\alpha, \beta, \gamma, \delta):=\prod_{i=1}^{n} d_{\operatorname{part}\left(\alpha_{i} \beta_{i} \gamma_{i} \delta_{i}\right)}^{*},
$$

which is a degree $n$ polynomial on $\left(\mathbb{R}^{q \times q} \otimes \mathbb{R}^{q \times q}\right)^{S_{q}}$. Then $\psi(\alpha, \beta, \gamma, \delta)=\psi\left(\alpha^{\prime}, \beta^{\prime}, \gamma^{\prime}, \delta^{\prime}\right)$ if and only if $(\alpha, \beta, \gamma, \delta)$ and $\left(\alpha^{\prime}, \beta^{\prime}, \gamma^{\prime}, \delta^{\prime}\right)$ belong to the same $H$-orbit on $\left([q]^{n}\right)^{4}$. So this gives a bijection between the set $Q$ of degree $n$ monomials expressed in the dual basis $D^{*}$ and the set of $H$-orbits on $\left([q]^{n}\right)^{4} \cong\left([q]^{4}\right)^{n}$. The function $\left([q]^{n}\right)^{4} \rightarrow \mathcal{C}_{4}$ with $\left(\alpha_{1}, \ldots, \alpha_{4}\right) \mapsto$ $\left\{\alpha_{1}, \ldots, \alpha_{4}\right\}$ then gives a surjective function $\omega: Q \rightarrow \Omega \backslash\{\{\varnothing\}\}$.

For any $\mu \in Q$, define

$$
K_{\mu}:=\sum_{\substack{d_{1}, \ldots, d_{n} \in D \\ d_{1}^{*} \cdots d_{n}^{*}=\mu}} \bigotimes_{j=1}^{n} d_{j}
$$

Lemma 1 For each $\omega \in \Omega: L_{\omega}=\sum_{\substack{\mu \in Q \\ \omega(\mu)=\omega}} K_{\mu}$.

Proof Choose $\alpha, \beta, \gamma, \delta \in[q]^{n}$. Then

$$
\begin{aligned}
\sum_{\substack{\mu \in Q \\
\omega(\mu)=\omega}}\left(K_{\mu}\right)_{(\alpha, \beta),(\gamma, \delta)} & =\sum_{\substack{\mu \in Q \\
\omega(\mu)=\omega}} \sum_{\begin{array}{c}
P_{1}, \ldots, P_{n} \in \Pi \\
d_{P_{1}}^{*} \cdots d_{P_{n}}^{*}=\mu
\end{array}}\left(\bigotimes_{i=1}^{n} d_{P_{i}}\right)_{\alpha, \beta, \gamma, \delta} \\
& =\sum_{\substack{\mu \in Q \\
\omega(\mu)=\omega}} \sum_{\begin{array}{l}
P_{1}, \ldots, P_{n} \in \Pi \\
d_{P_{1}}^{*} \cdots d_{P_{n}}^{*}=\mu
\end{array}} \prod_{i=1}^{n}\left(d_{P_{i}}\right)_{\alpha_{i}, \beta_{i}, \gamma_{i}, \delta_{i}}^{*} .
\end{aligned}
$$


Now the latter value is 1 if $\omega\left(d_{\operatorname{part}\left(\alpha_{1} \beta_{1} \gamma_{1} \delta_{1}\right)}^{*} \cdots d_{\text {part }\left(\alpha_{n} \beta_{n} \delta_{n} \gamma_{n}\right)}^{*}\right)=\omega$, and is 0 otherwise. So it is equal to $\left(L_{\omega}\right)_{(\alpha, \beta),(\gamma, \delta)}$.

By this lemma, it suffices to compute $v_{\tau}^{\top} K_{\mu} v_{\sigma}$ for each $\mu \in Q$. To this end, define the following degree $n$ polynomial on $W:=\left(\mathbb{R}^{q \times q} \otimes \mathbb{R}^{q \times q}\right)^{S_{q}}$ :

$$
p_{\tau, \sigma}:=\prod_{i=1}^{k} \sum_{\substack{\tau_{i}^{\prime} \sim \tau_{i} \\ \sigma_{i}^{\prime} \sim \sigma_{i}}} \sum_{c_{i}, c_{i}^{\prime} \in C_{\lambda_{i}}} \operatorname{sgn}\left(c_{i} c_{i}^{\prime}\right) \prod_{y \in Y\left(\lambda_{i}\right)} B_{i}\left(\tau_{i}^{\prime} c_{i}(y)\right) \otimes B_{i}\left(\sigma_{i}^{\prime} c_{i}^{\prime}(y)\right) .
$$

This polynomial can be computed (i.e., expressed as linear combination of monomials in $\left.B_{i}(j) \otimes B_{i}(h)\right)$ in time bounded by a polynomial in $n$ (Gijswijt [5], see Appendix 2 in Sect. 1 below).

Lemma $2 \sum_{\mu \in Q}\left(v_{\tau}^{\top} K_{\mu} v_{\sigma}\right) \mu=p_{\tau, \sigma}$.

Proof We can write for each $\mu \in Q$ :

$$
v_{\tau}^{\top} K_{\mu} v_{\sigma}=\left(v_{\tau} \otimes v_{\sigma}\right)\left(K_{\mu}\right),
$$

using the fact that $v_{\boldsymbol{\tau}}, v_{\boldsymbol{\sigma}} \in\left(\left(\mathbb{R}^{q \times q}\right)^{\otimes n}\right)^{*}$ and $K_{\mu} \in\left(\mathbb{R}^{q \times q}\right)^{\otimes n} \otimes\left(\mathbb{R}^{q \times q}\right)^{\otimes n}$. So it suffices to show

$$
\sum_{\mu \in Q}\left(v_{\tau} \otimes v_{\sigma}\right)\left(K_{\mu}\right) \mu=p_{\tau, \sigma} .
$$

Consider any $f=f_{1} \cdots f_{n}$ with $f_{j} \in W^{*}$ for $j=1, \ldots, n$. Then

$$
f=\sum_{\mu \in Q}\left(\bigotimes_{j=1}^{n} f_{j}\right)\left(K_{\mu}\right) \mu .
$$

Indeed,

$$
\begin{aligned}
\sum_{\mu \in Q}\left(\bigotimes_{j=1}^{n} f_{j}\right)\left(K_{\mu}\right) \mu & =\sum_{\substack{d_{1}, \ldots, d_{n} \in D \\
d_{1}^{*} \cdots d_{n}^{*}=\mu}}\left(\bigotimes_{j=1}^{n} f_{j}\right)\left(\bigotimes_{j=1}^{n} d_{j}\right) \mu \\
& =\sum_{d_{1}, \ldots, d_{n} \in D} \prod_{j=1}^{n} f_{j}\left(d_{j}\right) d_{j}^{*}=\prod_{j=1}^{n} \sum_{d \in D} f_{j}(d) d^{*} \\
& =\prod_{j=1}^{n} f_{j}=f .
\end{aligned}
$$

Applying (33) to each term $f$ of $p_{\tau, \sigma}$ as given by (30) we obtain (32), in view of (7) and (13).

So $v_{\tau}^{\top} K_{\mu} v_{\sigma}$ can be computed by expressing the polynomial $p_{\tau, \sigma}$ as linear combination of monomials $\mu \in Q$, which are products of linear functions in $D^{*}$. So it suffices to express each $B_{i}(j) \otimes B_{i}(h)$ as linear function into the basis $D^{*}$, that is, to calculate the numbers $\left(B_{i}(j) \otimes B_{i}(h)\right)\left(d_{P}\right)$ for all $i=1, \ldots, k, j, h=1, \ldots, m_{i}$, and $P \in \Pi$-see Appendix 3 (Sect. 1 below). 
We finally consider the entries in the row and column for $\emptyset$ in the matrix associated with $\lambda=((n),(),(),())$ (cf. Sect. 3.5). Trivially, $e_{\emptyset}^{\top} M(x) e_{\emptyset}=(M(x))_{\emptyset, \emptyset}=x(\emptyset)$, which is set to 1 in the optimization problem. Any $\tau \in W_{\lambda}$ is determined by the number $t$ of 2's in the row of the Young shape $Y((n))$. Then

$$
v_{\tau}=\sum_{\substack{u, w \in[q]^{n} \\ d_{H}(u, w)=t}} e_{(u, w)} \text { and hence } F v_{\tau}=\sum_{\substack{u, w \in[q]^{n} \\ d_{H}(u, w)=t}} e_{\{u, w\}} .
$$

Hence, as $\emptyset \cup\{u, w\}=\{u, w\}$,

$$
e_{\varnothing}^{\top} M(x) F v_{\tau}=\sum_{\substack{u, w \in[q]^{n} \\
d_{H}(u, w)=t}} x(\{u, w\})=\left(\begin{array}{l}
n \\
t
\end{array}\right) q^{n}(q-1)^{t} y(\omega),
$$

where $\omega$ is the $H$-orbit of $\mathcal{C}_{4}$ consisting of all pairs $\{\alpha, \beta\}$ with $d_{H}(\alpha, \beta)=t$.

Acknowledgements We are very grateful to Hans D. Mittelmann for his help in solving the larger semidefinite programming problems, and to the referee for helpful suggestions as to the presentation of the paper. The research leading to these results has received funding from the European Research Council under the European Union's Seventh Framework Programme (FP7/2007-2013)/ERC Grant Agreement nº 339109.

Open Access This article is distributed under the terms of the Creative Commons Attribution 4.0 International License (http://creativecommons.org/licenses/by/4.0/), which permits unrestricted use, distribution, and reproduction in any medium, provided you give appropriate credit to the original author(s) and the source, provide a link to the Creative Commons license, and indicate if changes were made.

\section{Appendix 1: The representative set $\left\{B_{1}, \ldots, B_{k}\right\}$ for the action of $S_{q}$ on $\mathbb{C}^{q \times q}$}

In this section we show that the matrix set $\left\{B_{1}, \ldots, B_{k}\right\}$ as given in (11) is representative for the natural action of $S_{q}$ on $\mathbb{C}^{q \times q}$. For $a \in \mathbb{C}^{q}$, let $\Delta_{a}$ be the $q \times q$ diagonal matrix with diagonal $a$, and let $\mathbf{1}$ be the all-one column vector in $\mathbb{C}^{q}$. Define

$$
\begin{aligned}
& V_{1,1}:=\left\{\lambda I_{q} \mid \lambda \in \mathbb{C}\right\}, \\
& V_{1,2}:=\left\{\lambda\left(J_{q}-I_{q}\right) \mid \lambda \in \mathbb{C}\right\}, \\
& V_{2,1}:=\left\{\Delta_{a} \mid a \in \mathbb{C}^{q}, a^{\top} \mathbf{1}=0\right\}, \\
& V_{2,2}:=\left\{a \mathbf{1}^{\top}-\mathbf{1} a^{\top} \mid a \in \mathbb{C}^{q}, a^{\top} \mathbf{1}=0\right\}, \\
& V_{2,3}:=\left\{a \mathbf{1}^{\top}+\mathbf{1} a^{\top}-2 \Delta_{a} \mid a \in \mathbb{C}^{q}, a^{\top} \mathbf{1}=0\right\}, \\
& V_{3,1}:=\left\{X \in \mathbb{C}^{q \times q} \mid X \text { skew-symmetric, } X \mathbf{1}=0\right\}, \\
& V_{4,1}:=\left\{X \in \mathbb{C}^{q \times q} \mid X \text { symmetric, } X \mathbf{1}=0, X_{i, i}=0 \text { for all } i \in[q]\right\} .
\end{aligned}
$$

Observe that each $V_{i, j}$ is $S_{q}$-stable, and that $V_{i, j}$ and $V_{i^{\prime}, j^{\prime}}$ are orthogonal whenever $(i, j) \neq$ $\left(i^{\prime}, j^{\prime}\right)$ (with respect to the inner product $X, Y \mapsto \operatorname{tr}\left(X^{*} Y\right)$ ). Moreover $\lambda I_{q} \mapsto \lambda\left(J_{q}-I_{q}\right)$ gives an $S_{q}$-isomorphism $V_{1,1} \rightarrow V_{1,2}, \Delta_{a} \mapsto a \mathbf{1}^{\top}-\mathbf{1} a^{\top}$ gives an $S_{q}$-isomorphism $V_{2,1} \rightarrow$ $V_{2,2}$, and $\Delta_{a} \mapsto a \mathbf{1}^{\top}+\mathbf{1} a^{\top}-2 \Delta_{a}$ gives an $S_{q}$-isomorphism $V_{2,1} \rightarrow V_{2,3}$.

Let $q \geq 4$. Then $\operatorname{dim}\left(V_{i, j}\right)>0$ for all $i, j$. Set, as before, $m_{1}=2, m_{2}=3, m_{3}=m_{4}=1$. Then $\sum_{i=1}^{4} m_{i}^{2}=15$, which is equal to the number of partitions of $\{1,2,3,4\}$, hence to the dimension of $\left(\mathbb{C}^{q \times q} \otimes \mathbb{C}^{q \times q}\right)^{S_{q}}$. This implies that the $V_{i, j}$ in fact form an orthogonal decomposition of $\mathbb{C}^{q \times q}$ into irreducible representations and that $V_{i, j}$ and $V_{i^{\prime}, j^{\prime}}$ are equivalent 
representations if and only if $i=i^{\prime}$ (as any further representation, or decomposition, or equivalence would yield that the sum of the squares of the multiplicities of the irreducible representations is strictly larger than 15 , contradicting the fact that $\Phi$ in (4) is bijective).

Now $B_{1,1}$ and $B_{1,2}$ are the elements of $V_{1,1}$ and $V_{1,2}$ with $\lambda=1$. Moreover, $B_{2,1}, B_{2,2}$, and $B_{2,3}$ are the elements of $V_{2,1}, V_{2,2}$, and $V_{2,3}$ with $a=e_{1}-e_{2}$. Finally, $B_{3,1}$ and $B_{4,1}$ are nonzero elements of $V_{3,1}$ and $V_{4,1}$ (they can be chosen arbitrarily). This implies that $\left\{B_{1}, \ldots, B_{4}\right\}$ is a representative matrix set.

If $q=3$, then $\operatorname{dim}\left(V_{4,1}\right)=0$, while the dimension of $\left(\mathbb{C}^{3 \times 3} \otimes \mathbb{C}^{3 \times 3}\right)^{S_{3}}$ is equal to the number of partitions of $\{1,2,3,4\}$ into at most 3 classes, which is $2^{2}+3^{2}+1^{2}=14$. If $q=2$, then moreover $\operatorname{dim}\left(V_{2,3}\right)=\operatorname{dim}\left(V_{3,1}\right)=0$, while the dimension of $\left(\mathbb{C}^{2 \times 2} \otimes \mathbb{C}^{2 \times 2}\right)^{S_{2}}$ is equal to the number of partitions of $\{1,2,3,4\}$ into at most 2 classes, which is $2^{2}+2^{2}=8$. Similarly as above, this implies that also for $q \leq 3, B_{1}, \ldots, B_{k}$ form a representative matrix set.

\section{Appendix 2: Computation of $p_{\tau, \sigma}$}

For any $n, m \in \mathbb{Z}_{+}, \lambda \vdash n$, and $\tau, \sigma \in T_{\lambda, m}$, define the polynomial $p_{\tau, \sigma} \in \mathbb{R}\left[x_{j, h} \mid j, h=\right.$ $1, \ldots, m]$ by

$$
p_{\tau, \sigma}(X):=\sum_{\substack{\tau^{\prime} \sim \tau \\ \sigma^{\prime} \sim \sigma}} \sum_{c, c^{\prime} \in C_{\lambda}} \operatorname{sgn}\left(c c^{\prime}\right) \prod_{y \in Y(\lambda)} x_{\tau^{\prime} c(y), \sigma^{\prime} c^{\prime}(y)},
$$

for $X=\left(x_{j, h}\right)_{j, h=1}^{m} \in \mathbb{R}^{m \times m}$.

Proposition 3 Expressing $p_{\tau, \sigma}$ as a linear combination of monomials can be done in polynomial time, for fixed $\mathrm{m}$.

Proof First observe that

$$
\begin{aligned}
p_{\tau, \sigma}(X)=\left|C_{\lambda}\right| \sum_{\substack{\tau^{\prime} \sim \tau \\
\sigma^{\prime} \sim \sigma}} \sum_{\substack{c \in C_{\lambda}\\
}} \operatorname{sgn}(c) \prod_{y \in Y_{\lambda}} x_{\tau^{\prime}(y), \sigma^{\prime} c(y)} \\
=\left|C_{\lambda}\right| \sum_{\substack{\tau^{\prime} \sim \tau \\
\sigma^{\prime} \sim \sigma}} \prod_{j=1}^{\lambda_{1}} \operatorname{det}\left(\left(x_{\tau^{\prime}(i, j), \sigma^{\prime}\left(i^{\prime}, j\right)}\right)_{i, i^{\prime}=1}^{\lambda_{j}^{*}}\right) .
\end{aligned}
$$

( $\lambda^{*}$ is the dual partition of $\lambda$; that is, $\lambda_{j}^{*}$ is the height of column $j$.)

For fixed $m$, when $n$ grows, there will be several columns of $Y(\lambda)$ that are the same both in $\tau^{\prime}$ and in $\sigma^{\prime}$. More precisely, for given $\tau^{\prime}, \sigma^{\prime}$ let the 'count function' $\kappa$ be defined as follows: for $t \in \mathbb{Z}_{+}$and $v, w \in[m]^{t}, \kappa(v, w)$ is the number of columns $j$ of height $t$ such that $\tau^{\prime}(i, j)=v_{i}$ and $\sigma^{\prime}(i, j)=w_{i}$ for all $i=1, \ldots, t$. Then for each $i \leq h:=\operatorname{height}(\lambda)$ and each $s \in[m]$ :

$$
\begin{aligned}
& \sum_{\substack{t=i \\
v, w \in[m]^{t} \\
v_{i}=s}}^{h} \kappa(v, w)=\text { number of } s \text { in row } i \text { of } \tau, \text { and } \\
& \sum_{t=i}^{h} \sum_{\substack{v, w \in[m]^{t} \\
w_{i}=s}} \kappa(v, w)=\text { number of } s \text { in row } i \text { of } \sigma .
\end{aligned}
$$


For any given function $\kappa: \bigcup_{i=1}^{h}[m]^{i} \times[m]^{i} \rightarrow \mathbb{Z}_{+}$satisfying (40), there are precisely

$$
\prod_{t=1}^{h} \frac{\left(\lambda_{t}-\lambda_{t+1}\right) !}{\prod_{v, w \in[m]^{t}} \kappa(v, w) !}
$$

pairs $\tau^{\prime} \sim \tau$ and $\sigma^{\prime} \sim \sigma$ having count function $\kappa$ (setting $\lambda_{h+1}:=0$ ). (Note that (40) implies $\lambda_{t}-\lambda_{t+1}=\sum_{v, w \in[m]^{t}} \kappa(v, w)$, for each $t$, so that for each $t$, the factor in (41) is a Newton multinomial coefficient.) Hence

$$
p_{\tau, \sigma}=\left|C_{\lambda}\right| \sum_{\kappa} \prod_{t=1}^{h}\left(\lambda_{t}-\lambda_{t+1}\right) ! \prod_{v, w \in[m]^{t}} \frac{\operatorname{det}\left(\left(x_{v(i), w\left(i^{\prime}\right)}\right)_{i, i^{\prime}=1}^{t}\right)^{\kappa(v, w)}}{\kappa(v, w) !},
$$

where $\kappa$ ranges over functions $\kappa: \bigcup_{t=1}^{h}\left([m]^{t} \times[m]^{t}\right) \rightarrow \mathbb{Z}_{+}$satisfying (40).

\section{Appendix 3: Expressing $B_{i}(j) \otimes B_{i}(h)$ into $d_{P}^{*}$}

Recall that each $B_{i}(j)$ is a linear function on $\mathbb{R}^{q \times q}$, and that each $d_{P}$ is an element of $\mathbb{R}^{q \times q} \otimes \mathbb{R}^{q \times q}$, where $P$ belongs to the set $\Pi$ of partitions of $\{1, \ldots, 4\}$ with at most $q$ classes. We express each $B_{i}(j) \otimes B_{i}(h)$ in the dual basis $B^{*}:=\left\{d_{P}^{*} \mid P \in \Pi\right\}$. The coefficient of $d_{P}^{*}$ is obtained by evaluating $\left(B_{i}(j) \otimes B_{i}(h)\right)\left(d_{P}\right)$. This is routine, but we display the expressions.

For this, denote any subset $X$ of $\{1, \ldots, 4\}$ by a string formed by the elements of $X$, and denote a partition $P$ of $\{1, \ldots, 4\}$ by a sequence of its classes (for instance, $d_{13,2,4}^{*}$ denotes the dual variable $d_{P}^{*}$ associated with partition $P=\{\{1,3\},\{2\},\{4\}\}$ of $\left.\{1,2,3,4\}\right)$. Then:

$$
\begin{aligned}
B_{1}(1) \otimes B_{1}(1)= & q d_{1234}^{*}+q(q-1) d_{12,34}^{*}, \\
B_{1}(1) \otimes B_{1}(2)= & q(q-1)\left(d_{123,4}^{*}+d_{124,3}^{*}+(q-2) d_{12,3,4}^{*}\right), \\
B_{1}(2) \otimes B_{1}(1)= & q(q-1)\left(d_{1,234}^{*}+d_{134,2}^{*}+(q-2) d_{1,2,34}^{*}\right), \\
B_{1}(2) \otimes B_{1}(2)= & q(q-1)\left(d_{13,24}^{*}+d_{14,23}^{*}+(q-2)\left(d_{13,2,4}^{*}+d_{14,2,3}^{*}+d_{1,23,4}^{*}\right.\right. \\
& \left.\left.+d_{1,24,3}^{*}+(q-3) d_{1,2,3,4}^{*}\right)\right) \\
B_{2}(1) \otimes B_{2}(1)= & 2 d_{1234}^{*}-2 d_{12,34}^{*}, \\
B_{2}(1) \otimes B_{2}(2)= & 2 q\left(d_{123,4}^{*}-d_{124,3}^{*}\right) \\
B_{2}(1) \otimes B_{2}(3)= & 2(q-2)\left(d_{124,3}^{*}+d_{123,4}^{*}-2 d_{12,3,4}^{*}\right), \\
B_{2}(2) \otimes B_{2}(1)= & 2 q\left(d_{134,2}^{*}-d_{1,234}^{*}\right), \\
B_{2}(2) \otimes B_{2}(2)= & 2 q\left(2 d_{13,24}^{*}-2 d_{14,23}^{*}+(q-2)\left(d_{13,2,4}^{*}-d_{14,2,3}^{*}-d_{1,23,4}^{*}+d_{1,24,3}^{*}\right)\right), \\
B_{2}(2) \otimes B_{2}(3)= & 2 q(q-2)\left(d_{13,2,4}^{*}+d_{14,2,3}^{*}-d_{1,23,4}^{*}-d_{1,24,3}^{*}\right), \\
B_{2}(3) \otimes B_{2}(1)= & 2(q-2)\left(d_{1,234}^{*}+d_{134,2}^{*}-2 d_{1,2,34}^{*}\right), \\
B_{2}(3) \otimes B_{2}(2)= & 2 q(q-2)\left(d_{13,2,4}^{*}-d_{14,2,3}^{*}+d_{1,23,4}^{*}-d_{1,24,3}^{*}\right), \\
B_{2}(3) \otimes B_{2}(3)= & 2(q-2)\left(2 d_{13,24}^{*}+2 d_{14,23}^{*}+(q-4)\left(d_{13,2,4}^{*}+d_{14,2,3}^{*}+d_{1,23,4}^{*}\right.\right. \\
& \left.\left.+d_{1,24,3}^{*}\right)-4(q-3) d_{1,2,3,4}^{*}\right) . \\
B_{3}(1) \otimes B_{3}(1)= & 6\left(d_{13,24}^{*}-d_{14,23}^{*}-d_{13,2,4}^{*}+d_{14,2,3}^{*}+d_{1,23,4}^{*}-d_{1,24,3}^{*}\right) . \\
B_{4}(1) \otimes B_{4}(1)= & 8\left(d_{13,24}^{*}+d_{14,23}^{*}-d_{13,2,4}^{*}-d_{14,2,3}^{*}-d_{1,23,4}^{*}-d_{1,24,3}^{*}\right)+16 d_{1,2,3,4}^{*} .
\end{aligned}
$$




\section{References}

1. Bogdanova G.T., Brouwer A.E., Kapralov S.N., Östergård P.R.J.: Error-correcting codes over an alphabet of four elements. Discret. Comput. Geom. 23, 333-342 (2001).

2. Bogdanova G.T., Östergård P.R.J.: Bounds on codes over an alphabet of five elements. Discret. Math. 240, 13-19 (2001).

3. Brouwer A.E.: Tables of code bounds, see http://www.win.tue.nl/ aeb/ (2015).

4. Delsarte P.: An algebraic approach to the association schemes of coding theory. Philips Research Reports Supplements 1973 No. 10, Philips Research Laboratories, Eindhoven (1973).

5. Gijswijt D.: Block diagonalization for algebra's associated with block codes. arXiv:0910.4515 (2014).

6. Gijswijt D.C., Mittelmann H.D., Schrijver A.: Semidefinite code bounds based on quadruple distances. IEEE Trans. Inf. Theory 58, 2697-2705 (2012).

7. Gijswijt D., Schrijver A., Tanaka H.: New upper bounds for nonbinary codes based on the Terwilliger algebra and semidefinite programming. J. Comb. Theory A 113, 1719-1731 (2006).

8. Sagan B.E.: The Symmetric Group: Representations, Combinatorial Algorithms, and Symmetric Functions, Graduate Texts in Mathematics, vol. 203. Springer, New York (2001).

9. Schrijver A.: New code upper bounds from the Terwilliger algebra and semidefinite programming. IEEE Trans. Inf. Theory 51, 2859-2866 (2005). 